\title{
Effect of a pathological scapular tilt after total shoulder arthroplasty
}

\author{
Marion Röthlisberger ${ }^{\mathrm{a}}$, Dominique P. Pioletti ${ }^{\mathrm{a}}$, Alain Farron ${ }^{\mathrm{b}}$ and Alexandre Terrier ${ }^{\mathrm{a} *}$ \\ ${ }^{a}$ Laboratory of Biomechanical Orthopedics, EPFL, Lausanne, Switzerland; ${ }^{b}$ University Hospital Center and University of Lausanne, \\ Lausanne, Switzerland
}

(Received 21 July 2011; final version received 9 January 2012)

\begin{abstract}
Total shoulder arthroplasty (TSA) is an accepted and most successfully used treatment for different shoulder pathologies. Different risk factors for the failure of the prosthesis are known. A pathological scapular orientation, observed in elderly people or in patients suffering from neuromuscular diseases, could be a cause of failure, which has not been investigated yet. To test this hypothesis, a numerical musculoskeletal model of the glenohumeral joint was used to compare two TSA cases: a reference normal case and a case with a pathological anterior tilt of the scapula. An active abduction of $150^{\circ}$ was simulated. Joint force, contact pattern, polyethylene and cement stress were evaluated for both cases. The pathological tilt slightly increased the joint force and the contact pressure, but also shifted the contact pattern. This eccentric contact increased the stress level within the polyethylene of the glenoid component and within the surrounding cement layer. This adverse effect occurred mainly during the first $60^{\circ}$ of abduction. Therefore, a pathological orientation of the scapula may increase the risk of a failure of the cement layer around the glenoid component. These preliminary numerical results should be confirmed by a clinical study.
\end{abstract}

Keywords: total shoulder arthroplasty; musculoskeletal model; scapular orientation

\section{Introduction}

Total shoulder arthroplasty (TSA) is a classical treatment for degenerative diseases of the glenohumeral joint. Although most patients are subjectively satisfied with the outcome after TSA, there are cases with unsatisfactory results after the implantation of the prosthesis (Deshmukh et al. 2005; Franta et al. 2007). Component malposition, glenohumeral malalignment and glenoid loosening are the most important technical issues in patients with poor results (Franta et al. 2007). One risk factor for the loosening of the glenoid component is an eccentric loading pattern, which might be due to a malposition of the components or an abnormal glenoid version (Matsen et al. 2008). Cadaveric experiments (Nyffeler et al. 2006; Shapiro et al. 2007) as well as numerical models (Farron et al. 2006; Terrier et al. 2009b) had shown an increased risk for a loosening of the glenoid component in cases where the glenoid is unnaturally oriented. It is, however, not yet known to what extend an altered position of the scapula can influence the outcome after TSA.

The orientation of the scapula is individually different (Borstad and Ludewig 2002) and can be altered in older subjects (Culham and Peat 1993), patients with different pain syndromes (Helgadottir et al. 2010) or neuromuscular disorders (Pandya et al. 2008). Due to osteoporosis (De Smet et al. 1988), elderly patients often show an increased thoracic kyphosis, which lead to an increased anterior tilt of the scapula (Culham and Peat 1993). An imbalance of different shoulder muscles may also lead to an altered position of the scapula (Pandya et al. 2008; Helgadottir et al. 2010). This changed position of the scapula may induce an abnormal mechanical loading of the glenoid component. This may eventually lead to a higher risk of aseptic loosening of the glenoid component, which remains the major complication after anatomical TSA (Matsen et al. 2008).

Therefore, the aim of this study was to test whether an altered position of the scapula can preclude the survival of an anatomic TSA. Two cases were analysed and compared using a numerical musculoskeletal model of the shoulder: a reference case with a normal orientation of the scapula and a pathological case with an increased anterior tilt of the scapula.

\section{Methods}

A numerical musculoskeletal model of the shoulder was extended to compare normal and pathological orientations of the scapula (Terrier et al. 2005, 2007, 2008, 2009a, 2009b). The model contains the scapula and the proximal part of the humerus, which were reconstructed from CT scans of a cadaveric shoulder without any sign of pathologies. Six glenohumeral muscles were implemented in the model, namely the middle, anterior and posterior part of the deltoid, the supraspinatus, subscapularis and infraspinatus combined with teres minor. A dissection of

\footnotetext{
*Corresponding author. Email: alexandre.terrier@epfl.ch

(C) 2013 Taylor \& Francis
} 
the same cadaveric shoulder provided the origins and insertions of these muscles (Buchler et al. 2002). Muscles contained two parts: a passive part that wraps around the humerus and an active part that transmits the muscular force, providing the two functions of the shoulder muscles: stabilisation and motion. The contact force of the wrapping muscles and the glenohumeral contact force counterbalanced the weight of the fully extended arm $(37.5 \mathrm{~N})$ plus an additionally load in the hand $(10 \mathrm{~N})$ and provided the stability of the joint. This method allowed the natural translation of the humeral head within the glenoid fossa during motion.

An active abduction from $0^{\circ}$ to $150^{\circ}$ in the plane of the scapula was performed by a contraction of the glenohumeral muscles. As described in detail earlier (Terrier et al. 2007), a custom-made algorithm was used to determine the muscle forces needed for the movement of the humerus. The middle deltoid was defined as the main abductor muscle and a shortening of this muscle was imposed. The induced force was calculated and the forces of other five muscles were assumed to be a constant ratio of this force. Muscular force ratios were derived from physiological cross-sectional area and electromyography as initially proposed by Poppen and Walker (1978).

Two different motions of the scapula were performed and compared: a normal motion of the scapula (reference case) and a pathological motion (pathological case) of the scapula. For the reference case, the scapula was continuously rotated during the humeral abduction, assuming linear relationship with the total arm elevation, estimated from in vivo measurements (Borstad and Ludewig 2002). From $0^{\circ}$ to $150^{\circ}$ of abduction, the scapula rotated upward by $50^{\circ}$, rotated internally by $15^{\circ}$ and tilted anteriorly by $5^{\circ}$. For the pathological case, a constant offset of $10^{\circ}$ of anterior tilt was added during the whole range of abduction (Figure 1).

The Aequalis anatomical shoulder prosthesis (Tornier Inc., Edina, MN, USA) was inserted into the finite element model by a senior shoulder surgeon according to manufacturer's recommendations. The glenoid component axis was set coincidental to the glenoid centre line. A spherical bone resection with minimal subchondral bone removal was performed, and the keeled glenoid component was adjusted for the best support on the cortical wall. The cement thickness around the glenoid component was $0.5 \mathrm{~mm}$. The humeral component was placed to replicate the natural articular surface as closely as possible. The polyethylene glenoid component had a radius of curvature of $30 \mathrm{~mm}$ and the diameter of the metallic humeral head was $48 \mathrm{~mm}$. Polyethylene $(E=500 \mathrm{MPa}, \quad \nu=0.4)$ and cement $(E=2000 \mathrm{MPa}$, $\nu=0.3)$ were assumed linear elastic, while metallic parts were considered rigid. Bone was a linear elastic material with non-homogeneous mechanical properties derived from the Hounsfield number of the CT images.

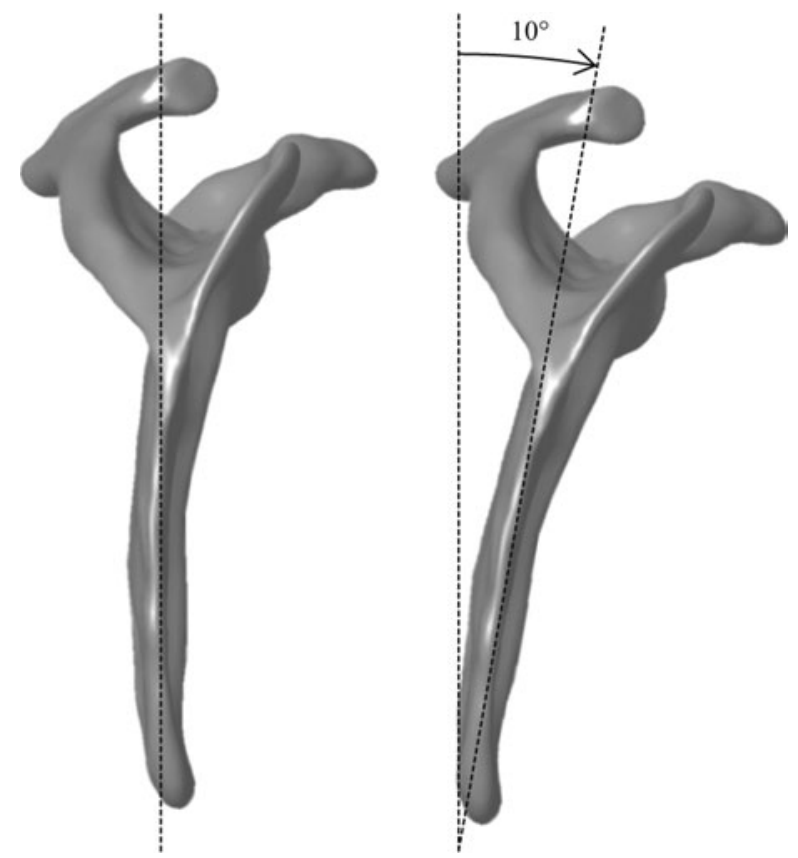

Figure 1. Orientation of scapula: left, reference case; right, pathological case.

The cement and polyethylene component were discretised with linear hexahedral elements and the bones with quadratic tetrahedral elements (Terrier et al. 2005). Totally, 10,400 elements for the cement and 2100 elements for the polyethylene component were used.

We analysed the glenohumeral joint contact force, the contact pressure pattern on the glenoid component, the von Mises stress within the polyethylene and the maximum principal (tensile) stress within the cement mantle. The volumetric distribution of the stress was evaluated in the polyethylene and in the cement. The analysis was carried out with Abaqus v6.9 (www.simulia.com).

\section{Results}

For both cases, the glenohumeral contact force increased during abduction up to approximately the horizontal position of the arm and decreased thereafter. The glenohumeral contact force was higher for the pathological case than for the reference case during the entire range of abduction, but especially at the beginning of the movement (Table 1). The maximal contact force reached $127.6 \%$ body weight $(\mathrm{BW})$ at $80^{\circ}$ of abduction for the reference case, and $133.3 \% \mathrm{BW}$ at $90^{\circ}$ of abduction for the pathological case, corresponding to an increase of $4.5 \%$.

The same trend as for the contact force was observed for the contact pressure (Table 1). It was indeed higher for the pathological case during the entire range of abduction. The maximal contact pressure occurred at $70^{\circ}$ of abduction for both cases. It was $22.8 \mathrm{MPa}$ for the reference case and 
Table 1. Relative differences $(\%)$ between the pathological and reference case $(<0$, larger values for reference case; $>0$, larger values for pathological case).

\begin{tabular}{lrrrrr}
\hline Abduction angle $\left({ }^{\circ}\right)$ & \multicolumn{1}{c}{5} & \multicolumn{1}{c}{30} & \multicolumn{1}{c}{60} & 90 & 120 \\
\hline Contact force (\%) & 22.7 & 14.5 & 11.8 & 4.4 & 1.2 \\
Contact pressure (\%) & -1.1 & 12.6 & 4.9 & 2.7 & 2.0 \\
Polyethylene stress (\%) & -8.4 & 33.2 & 6.6 & 3.6 & -6.1 \\
Cement stress (\%) & 28.5 & 9.9 & -1.1 & 8.0 & 13.4 \\
\hline
\end{tabular}

23.6 MPa for the pathological case, corresponding to an increase of $4.3 \%$. The contact pressure pattern on the glenoid surface followed an up and down movement during abduction (Figure 2). For the pathological case, the contact pattern was displaced towards the posterior border of the glenoid component during the first $60^{\circ}$ of abduction.

Within the polyethylene, the maximal stress reached $14.5 \mathrm{MPa}$ at $90^{\circ}$ of abduction for the reference case, and $16 \mathrm{MPa}$ at $30^{\circ}$ for the pathological case (Table 1). The volume of polyethylene with a stress value above $10 \mathrm{MPa}$ was higher for the pathological case during the entire abduction (Figure 3). The largest volume above $10 \mathrm{MPa}$ was observed at $60^{\circ}$ of abduction, corresponding to $2.8 \%$ of the total polyethylene volume for the reference case and $3.4 \%$ for the pathological case.

Within the cement surrounding the glenoid component, the stress was higher for the pathological case, except at $60^{\circ}$ of abduction, where stress was almost the same (Table 1). The maximal stress was $9.2 \mathrm{MPa}$ for the reference case and $9.1 \mathrm{MPa}$ for the pathological case, both at $60^{\circ}$ of abduction.

The pathological anterior tilt produced a higher volume of cement with a stress above $5 \mathrm{MPa}$ (Figure 4). The most important difference between reference and pathological cases occurred at $30^{\circ}$ and $60^{\circ}$ of abduction. At $30^{\circ}$ of abduction, $0.8 \%\left(5.6 \mathrm{~mm}^{3}\right)$ of the total volume in the pathological case and $1.7 \%\left(12.9 \mathrm{~mm}^{3}\right)$ in the healthy case were above a stress level of $5 \mathrm{MPa}$, which means an increase of $130 \%$ for the pathological case. At $60^{\circ}$ of abduction, $2.9 \%\left(21.7 \mathrm{~mm}^{3}\right)$ of the total volume for the reference case and $4.1 \%\left(30.6 \mathrm{~mm}^{3}\right)$ for the pathological case were above this stress level, which means an increase of $41 \%$ for the pathological case.

\section{Discussion}

The failure of the glenoid component is the most common complication after TSA (Matsen et al. 2008). There are different causes, but the damaging of the cement seems to be critical (Sarah et al. 2010). In this study, we tested the hypothesis that a pathological anterior tilt of the scapula can increase the failure risk of the glenoid component after TSA. We indeed predicted an increase in the volume of cement above a critical stress value of $5 \mathrm{MPa}$ for the pathological case. The position of the scapula and thus of the glenoid indeed seems to be an important factor influencing the survival rate after TSA. Studies that examined the effect of a retroversion or inclination of the glenoid on the implant survival found an increased risk of a loosening of the prosthesis (Farron et al. 2006; Nyffeler et al. 2006; Shapiro et al. 2007; Terrier et al. 2009b), which is in agreement with the findings of this study.

For the reference case, predictions were in agreement with previous publications. The maximal contact force $(127.3 \% \mathrm{BW})$ was in the same range as the value reported by Poppen and Walker (1978; 140\% BW), who also simulated abduction with $1 \mathrm{~kg}$ in the hand. Within cement (Lacroix et al. 2000; Murphy et al. 2001; Gupta et al. 2004) and polyethylene (Swieszkowski et al. 2003; Gupta et al. 2004), stress was also comparable with other finite element studies.

A $10^{\circ}$ increased anterior tilt of the scapula resulted in a slightly higher contact force and contact pressure,
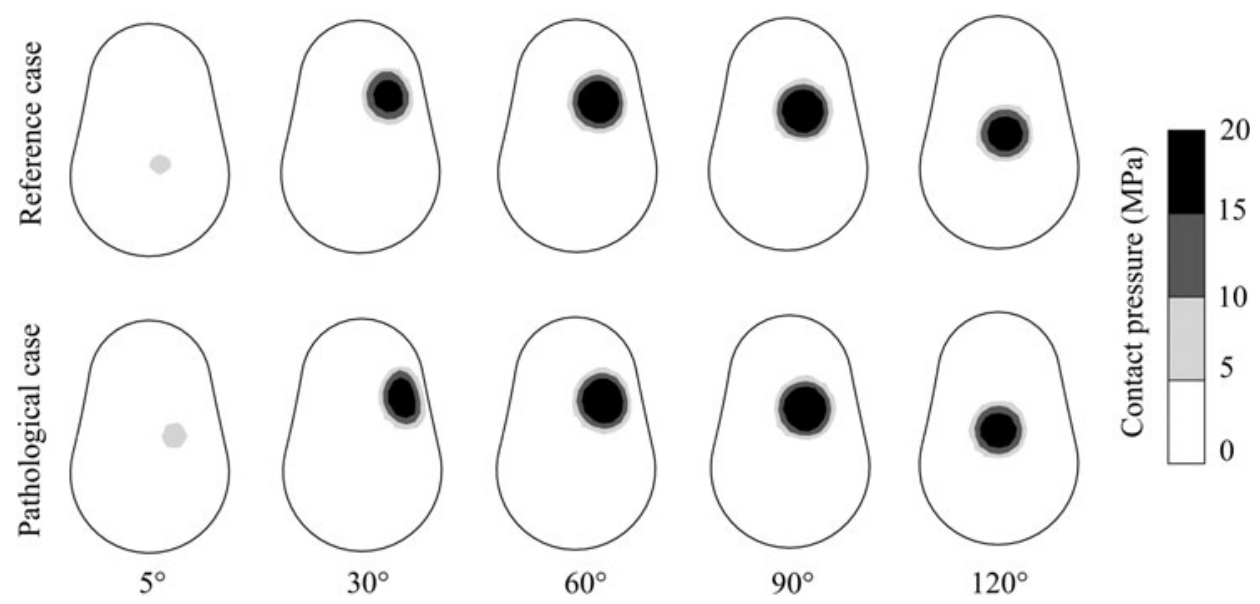

Figure 2. Patterns of contact pressure during humeral abduction (grey scale corresponding to 5, 10, 15 and $20 \mathrm{MPa}$ ). 


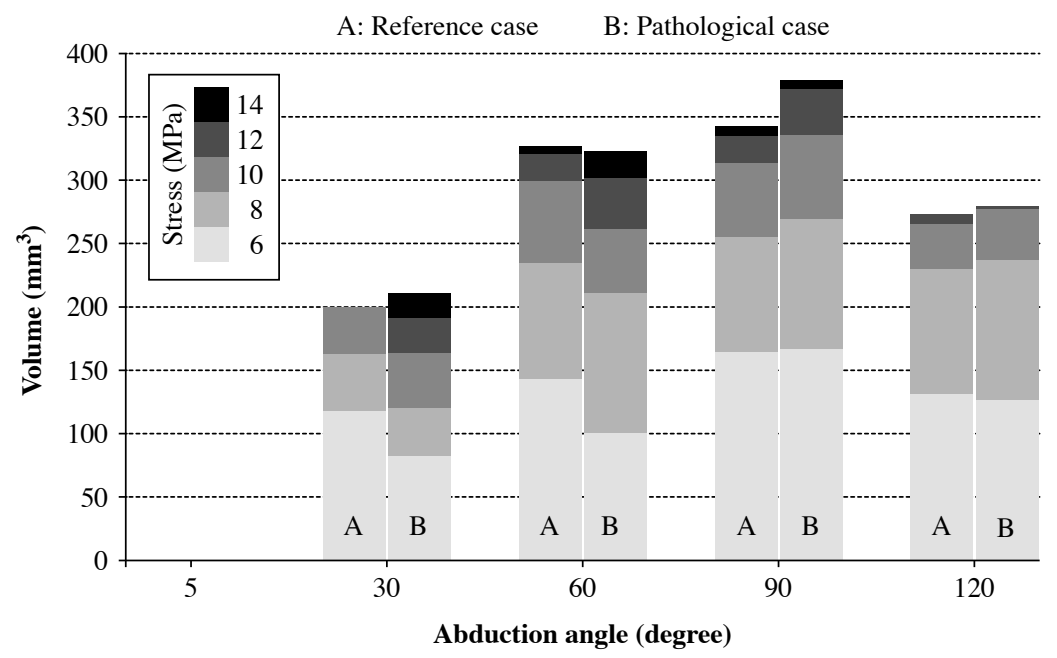

Figure 3. Volumetric distribution of the polyethylene stress for the reference case (A) and the pathological case (B).

an eccentric contact pattern, as well as higher cement and polyethylene stress mainly during the first $60^{\circ}$ of abduction. Since the arm is mainly below this abduction angle during the activities of daily living (Coley et al. 2008), we can expect a higher risk of aseptic loosening of the glenoid component. This can be related to the cement stress increase, and also to the eccentric contact pattern associated with the well-known rocking horse phenomenon (Matsen et al. 2008).

We analysed cement stress with the maximal principal stress invariant since tensile stress is the most critical parameter for the failure of the cement (Sarah et al. 2010). This stress quantity is associated with fatigue failure of about $5 \mathrm{MPa}$ (Lacroix et al. 2000). Our results showed that the pathological case induced an important increase in the volume of cement above this level, which can be associated with an increase in micro-cracks in the cement and eventually to a higher implant loosening occurrence. For the polyethylene, since the yield strength lies above $20 \mathrm{MPa}$ (Pruitt 2005), we would not exclude an important effect of an increased anterior scapular tilt on its damage. It was critical to account for the local non-homogeneous properties of bone to estimate correctly the cement and polyethylene stress.

The motion and position of the scapula differ between the subjects (Borstad and Ludewig 2002) and can be altered in older subjects (Culham and Peat 1993) and patients with disorders (Borstad and Ludewig 2002; Pandya et al. 2008; Helgadottir et al. 2010). A correlation between the trunk position and the scapular orientation (Kebaetse et al. 1999; Finley and Lee 2003) as well as between the age and the scapular orientation (Endo et al. 2004) was described in the literature. Elderly people often have an altered posture of the trunk (Fon et al. 1980;

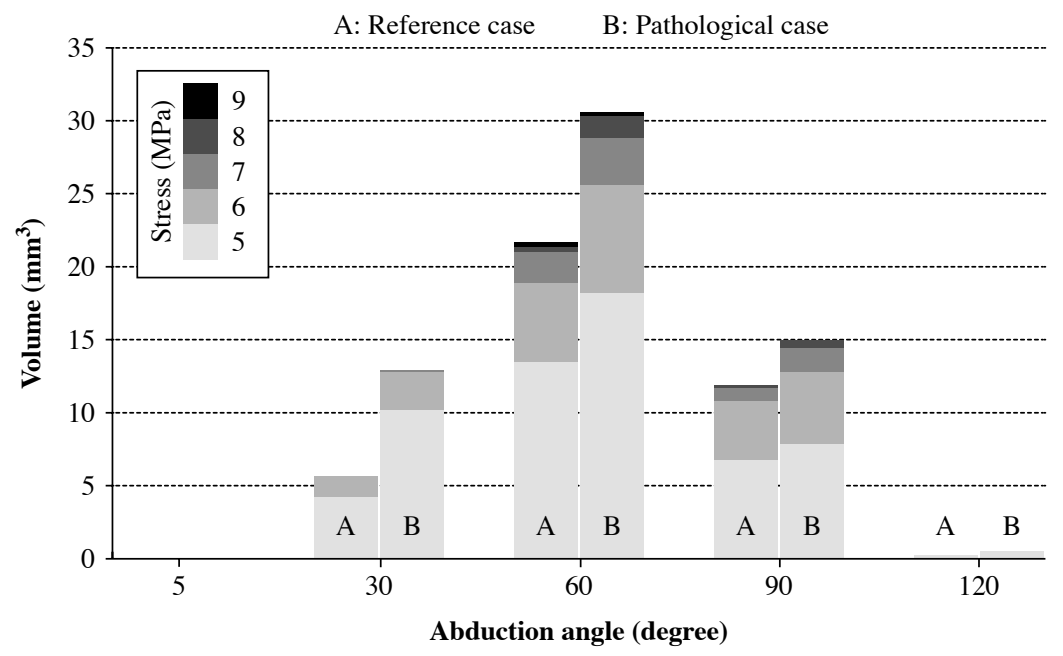

Figure 4. Volumetric distribution of the cement stress for the reference case (A) and the pathological case (B). 
Kauffman 1987; Culham and Peat 1993). These subjects frequently show an increased thoracic kyphosis, which may lead to an increased anterior tilt of the scapula (Culham and Peat 1993). It is known that older patients are at higher risk for an earlier failure of the TSA, but the exact reasons for this observation are unclear (Kepler et al. 2009). Thus, we simulated a $10^{\circ}$ increased anterior tilt of the scapula to approximate the altered position of the scapula in these patient groups.

The outcome of TSA is dependent on many different factors (Skirving 1999; Iannotti and Norris 2003). It is obvious that this study is a large simplification of the situation in patients. In the present parametric study, the orientation of the scapula during the abduction movement was the only varying parameter. In addition, only one movement was simulated. We have chosen abduction in the scapular plane because it is the most natural loaded movement of the shoulder, and thus the most reported movement in shoulder biomechanics. We, however, assume that testing other (more unfavourable) movements would lead to the same conclusion. Several studies have reported an important variability in the motion of the scapula. In the paper of Borstad and Ludewig, the 95\% confidence interval for anterior tipping (at $100^{\circ}$ of scapular abduction) was between $3.5^{\circ}$ and $9.6^{\circ}$ for 26 subjects. In our study, we have nearly tripled this average value (from about $5^{\circ}$ to $15^{\circ}$ ). Individual differences (in the remaining $5 \%$ ) may have larger effect than the additional $10^{\circ}$ tested in our comparison, but when we focus on the $95 \%$ of the population, we can reasonably consider a statistical effect. Therefore, we can assume that this theoretical prediction should be clinically observable. Although this theoretical study confirmed this hypothesis, we do not pretend to bring a definitive answer, but rather raised the relevance for a clinical trial. If this theoretical prediction is clinically confirmed, the present computer model could be further used to propose and test solution.

\section{Conclusion}

This study was the first approach in the evaluation of the effects of an altered motion of the scapula on long-term lifespan of anatomical TSA. Our results suggest that an increased anterior scapular tilt could induce a higher risk of glenoid component loosening, but this preliminary conclusion should be confirmed by a clinical study.

\section{Acknowledgements}

This study was supported by the Swiss National Science Foundation (K-32K1_122512) and by Tornier Inc., Edina, MN.

\section{References}

Borstad JD, Ludewig PM. 2002. Comparison of scapular kinematics between elevation and lowering of the arm in the scapular plane. Clin Biomech (Bristol, Avon). 17:650-659.
Buchler P, Ramaniraka NA, Rakotomanana LR, Iannotti JP, Farron A. 2002. A finite element model of the shoulder: application to the comparison of normal and osteoarthritic joints. Clin Biomech (Bristol, Avon). 17:630-639.

Coley B, Jolles BM, Farron A, Aminian K. 2008. Arm position during daily activity. Gait Posture. 28:581-587.

Culham E, Peat M. 1993. Functional anatomy of the shoulder complex. J Orthop Sports Phys Ther. 18:342-350.

De Smet AA, Robinson RG, Johnson BE, Lukert BP. 1988. Spinal compression fractures in osteoporotic women: patterns and relationship to hyperkyphosis. Radiology. 166: 497-500.

Deshmukh AV, Koris M, Zurakowski D, Thornhill TS. 2005. Total shoulder arthroplasty: long-term survivorship, functional outcome, and quality of life. J Shoulder Elbow Surg. 14:471-479.

Endo K, Yukata K, Yasui N. 2004. Influence of age on scapulothoracic orientation. Clin Biomech (Bristol, Avon). 19: 1009-1013.

Farron A, Terrier A, Buchler P. 2006. Risks of loosening of a prosthetic glenoid implanted in retroversion. J Shoulder Elbow Surg. 15:521-526.

Finley MA, Lee RY. 2003. Effect of sitting posture on 3-dimensional scapular kinematics measured by skinmounted electromagnetic tracking sensors. Arch Phys Med Rehabil. 84:563-568.

Fon GT, Pitt MJ, Thies AC, Jr. 1980. Thoracic kyphosis: range in normal subjects. AJR Am J Roentgenol. 134:979-983.

Franta AK, Lenters TR, Mounce D, Neradilek B, Matsen FA, 3rd. 2007. The complex characteristics of 282 unsatisfactory shoulder arthroplasties. J Shoulder Elbow Surg. 16:555-562.

Gupta S, van der Helm FC, van Keulen F. 2004. Stress analysis of cemented glenoid prostheses in total shoulder arthroplasty. J Biomech. 37:1777-1786.

Helgadottir H, Kristjansson E, Mottram S, Karduna AR, Jonsson H, Jr. 2010. Altered scapular orientation during arm elevation in patients with insidious onset neck pain and whiplash-associated disorder. J Orthop Sports Phys Ther. 40: 784-791.

Iannotti JP, Norris TR. 2003. Influence of preoperative factors on outcome of shoulder arthroplasty for glenohumeral osteoarthritis. J Bone Joint Surg Am. 85:251-258.

Kauffman T. 1987. Posture and age. Topics Geriatric Rehabil. 2:13-28.

Kebaetse M, McClure P, Pratt NA. 1999. Thoracic position effect on shoulder range of motion, strength, and three-dimensional scapular kinematics. Arch Phys Med Rehabil. 80:945-950.

Kepler CK, Nho SJ, Ala OL, Craig EV, Wright TM, Warren RF. 2009. Comparison of early and delayed failed total shoulder arthroplasty. Acta Orthop Belg. 75:297-305.

Lacroix D, Murphy LA, Prendergast PJ. 2000. Threedimensional finite element analysis of glenoid replacement prostheses: a comparison of keeled and pegged anchorage systems. J Biomech Eng. 122:430-436.

Matsen FA, 3rd, Clinton J, Lynch J, Bertelsen A, Richardson ML. 2008. Glenoid component failure in total shoulder arthroplasty. J Bone Joint Surg Am. 90:885-896.

Murphy LA, Prendergast PJ, Resch H. 2001. Structural analysis of an offset-keel design glenoid component compared with a center-keel design. J Shoulder Elbow Surg. 10:568-579.

Nyffeler RW, Sheikh R, Atkinson TS, Jacob HA, Favre P, Gerber C. 2006. Effects of glenoid component version on humeral head displacement and joint reaction forces: an experimental study. J Shoulder Elbow Surg. 15:625-629. 
Pandya S, King WM, Tawil R. 2008. Facioscapulohumeral dystrophy. Phys Ther. 88:105-113.

Poppen NK, Walker PS. 1978. Forces at the glenohumeral joint in abduction. Clin Orthop Relat Res 165-170.

Pruitt LA. 2005. Deformation, yielding, fracture and fatigue behavior of conventional and highly cross-linked ultra high molecular weight polyethylene. Biomaterials. 26:905-915.

Sarah J, Sanjay G, Sanjay S, Carolyn A, Emery R, Andrew A, Ulrich H. 2010. Failure mechanism of the all-polyethylene glenoid implant. J Biomech. 43:714-719.

Shapiro TA, McGarry MH, Gupta R, Lee YS, Lee TQ. 2007. Biomechanical effects of glenoid retroversion in total shoulder arthroplasty. J Shoulder Elbow Surg. 16:S90-S95.

Skirving AP. 1999. Total shoulder arthroplasty - current problems and possible solutions. J Orthop Sci. 4:42-53.

Swieszkowski W, Bednarz P, Prendergast PJ. 2003. Contact stresses in the glenoid component in total shoulder arthroplasty. Proc Inst Mech Eng H. 217:49-57.
Terrier A, Buchler P, Farron A. 2005. Bone-cement interface of the glenoid component: stress analysis for varying cement thickness. Clin Biomech (Bristol, Avon). 20:710-717.

Terrier A, Merlini F, Pioletti DP, Farron A. 2009a. Comparison of polyethylene wear in anatomical and reversed shoulder prostheses. J Bone Joint Surg Br. 91:977-982.

Terrier A, Merlini F, Pioletti DP, Farron A. 2009b. Total shoulder arthroplasty: downward inclination of the glenoid component to balance supraspinatus deficiency. J Shoulder Elbow Surg. 18:360-365.

Terrier A, Reist A, Merlini F, Farron A. 2008. Simulated joint and muscle forces in reversed and anatomic shoulder prostheses. J Bone Joint Surg Br. 90:751-756.

Terrier A, Reist A, Vogel A, Farron A. 2007. Effect of supraspinatus deficiency on humerus translation and glenohumeral contact force during abduction. Clin Biomech (Bristol, Avon). 22:645-651. 